\title{
FOURIER TRANSFORMS OF DINI-LIPSCHITZ FUNCTIONS
}

\author{
M.S. YOUNIS \\ Department of Mathematics \\ Yarmouk University, Irbid, Jordan \\ (Received October 29, 1984
}

ABSTRACT. It is well known that if Lipschitz conditions of a certain order are imposed on a function $f(x)$, then these conditions affect considerably the absolute convergence of the Fourier series and Fourier transforms of $f$. In general, if $f(x)$ belongs to a certain function class, then the Lipschitz conditions have bearing as to the dual space to which the Fourier coefficients and transforms of $f(x)$ belong. In the present work we do study the same phenomena for the wider Dini-Lipschitz class as well as for some other allied classes of functions.

KEY WORDS AND PHRASES. Dini-Lipschitz functions, Fourier series, Fourier transforms. 1980 AMS SUBJECT CLASSIFICATION CODE. $42 \mathrm{~A}, 44$.

1. INTRODUCTION.

TITCHMARSH ([1] Theorems 84,85) proved that if $f(x)$ belongs to the Lipschitz class Lip $(\alpha, p)$ in the $L^{p}$ norm on the real Line $R$, then its Fourier transform $\hat{f}$ belongs to $L^{\beta}(R)$ for

$$
\frac{p}{p+\alpha p-1}<\beta \leq p^{\prime}=\frac{p}{p-1}
$$

$0<\alpha \leq 1,1<\mathrm{p} \leq 2$

In [2] and [3] we extended Titchmarsh's Theorems to heigher differences and to functions of several variables on $R^{n}$ and $T^{n}$ where $T^{n}$ is the $n$ dimensional torus group.

In this paper we try, among other things, to explore the validity of those theorems in case of functions of the wider Dini-Lipschitz class on various groups.

2. DEFINITIONS AND NOTATIONS.

In the sequel, $R$ will denote the real line, $R^{n}$ stands for the $n$-dimensional Euclidian space, $T$ and $T^{n}$ denote the circle group $[0,2 \pi]$ and the n-dimensional torus respectively. $L^{p}$ consists of all equivalent classes of functions such that

$$
\|f\|_{p}=\left[\int|f|^{p} d x\right]^{\frac{1}{p}}<\infty .
$$


DEF [NITION 2.1.

Let $f(x) \& L^{p}(R)$. The Fourier Transform $\hat{f}$ of $f$ is defined by

$$
\hat{f}(u)=\frac{1}{2 \pi} \int_{R} f(x) e^{-i x u} d x .
$$

If $f(x) \in L_{L}^{P}(T)$ however, its Fourier series is given by

$$
f(x)=|n| \sum^{\infty} c_{n} e^{-i u x}
$$

With the usual modifications of these two definitions for functions of several variables in $L^{P}\left(R^{n}\right)$ and $L^{p}\left(T^{n}\right)$ respectively.

DEFINITION 2.2.

Let $f(x) \& \quad L^{p}(R)$ or $L^{p}(T)$.

Then the integral modulus of continuity $w_{p}(h, f)$ is defined by

$$
w_{p}(h, f)=\sup _{|x| \leq h}\|f(x+h)-f(x)\|_{p}
$$

For $p=\infty$ we write

$$
w(h, f)=\operatorname{Sup}_{|x|<h} \| f(x+h)-f(x)||
$$

DEFINITION 2.3 .

Let

$$
\begin{aligned}
& w_{p}(h, f)=0\left(h^{\alpha}\right) \\
& o\left(h^{\alpha}\right)
\end{aligned}
$$

as $h+0$. Then we say that $f(x)$ belongs to the Lipschitz class $\operatorname{Lip}(\alpha, p)$ or to the Little lipschitz class $1 \mathrm{i}(\alpha, p)$ respectively.

DEFINITION 2.4 .

Krovokin ([4], p. 65) defines the Dini-Lipschitz class as those functions such that

$$
\operatorname{Lim}_{h \rightarrow 0} w(h, f) \log \left(\frac{1}{h}\right) \rightarrow 0 .
$$

Equivalently one could write

$$
\operatorname{Lim}_{h \rightarrow 0} w(h, f)=0\left[\log \left(\frac{1}{h}\right)\right]^{-1}
$$

For functions in $L^{p}$ spaces. We can define the Dini-Lipschitz classes as those for which

$$
\operatorname{Lim}_{h \rightarrow 0} w_{p}(h, f)=o\left[\log \left(\frac{1}{h}\right)\right]^{-1}
$$


A still further extension is possible if we write

$$
\operatorname{Lim}_{h \rightarrow 0} w_{p}(h, f)=o\left[\log \left(\frac{1}{h}\right)\right]^{-\gamma}
$$

for some $\gamma$.

3. FOURIER TRANSFORMS OF DINI-LIPSCHITZ FUNCTIONS.

Our aim is to show that the conclusion of Titchmarsh's Theorem 84 [1, p. 115] does not hold for the Dini-Lipschitz functions in $L^{p}(R)$ and that all what we can say about their Fourier transforms $\hat{f}$ is that $\hat{f}$ belongs to $L^{p^{\prime}}(R)$ where $\frac{1}{p}+\frac{1}{p^{\prime}}=1$. Thus we prove the following.

THEOREM 3.1 .

Let $f(x)$ belong to the Dini-Lipschitz class in $L^{p}(R)$. Then $\hat{f}$ belongs to $L^{P^{\prime}}$, $1<\mathrm{p} \leq 2$

PROOF. Notice that

$$
w_{p}(h, f)=o\left[\log \left(\frac{1}{h}\right)\right]^{-1}
$$

is equivalent to

$$
\int_{R}\|f(x+h)-f(x)\|^{p} d x=0\left[\log \left(\frac{1}{h}\right)\right]^{-p}
$$

Thus by taking the Fourier Transform of $|f(x+h)-f(x)|$, applying the HausdorffYoung inequality, and following Titchmarsh's proof we arrive at

$$
\int_{0}^{\frac{1}{h}}|u h \hat{f}|^{p^{\prime}} d u=o\left[\log \left(\frac{1}{h}\right)\right]^{-p^{\prime}}
$$

and hence

$$
\int_{0}^{\frac{1}{h}}|u \hat{f}|^{p^{\prime}} d u=0\left[h \log \left(\frac{1}{h}\right)\right]^{-p^{\prime}}
$$

Let

$$
\phi(\mathrm{x})=\int_{1}^{\mathrm{x}}|\mathrm{u} \hat{\mathrm{f}}|^{\beta} \mathrm{du}
$$

Then for $B \leq \mathrm{p}^{\prime}$ and by Holder inequality we obtain

$$
\begin{aligned}
& \left.\dot{\varphi}(\mathrm{X})=0\left[\mathrm{x}^{-1} \log (\mathrm{X})\right]^{\beta}[\mathrm{X}]\right]^{1-\beta}+\frac{\beta}{\mathrm{p}} \\
& =0[\log (\mathrm{X})]^{-\beta} \mathrm{x}^{1+\frac{\beta}{p}}
\end{aligned}
$$

which yields

$$
\int_{1}^{x}|\hat{f}|^{\beta} d u=o[\log x]^{-\beta} x^{1-\beta}+\frac{\beta}{p}
$$


For the right hand side of the last estimate to be bounded as $\mathrm{X} \rightarrow \infty$ we must have

$$
1-\beta+\frac{\beta}{p} \leq 0
$$

and

$$
-\beta<-1 \text { i.e. } 1<\beta
$$

which is always the case in our situation.

The first condition, however, gives

$$
\begin{aligned}
& p-\beta p+p \leq 0 \\
& \frac{p}{p-1}=p^{\prime} \leq B
\end{aligned}
$$

The case $p^{\prime} \leq B$ is rejected of course and we are left with $B=p^{\prime}$ which indicates that, in contrast to the Lipschitz functions, the imposition of DiniLipschitz conditions on our functions does not improve upon the conclusion of the Hausdorff-Young theorem and the proof is complete.

We remark at this point that if we employ the condition

$$
w_{p}(h, f)=o\left[\log \left(\frac{1}{h}\right)\right]^{-\gamma}
$$

in theorem 3.1 we obtain the two conditions.

$$
1-\beta+\frac{\beta}{p} \leq 0
$$

and

$$
-\gamma \beta<-1
$$

The first yields the previous conclusion $B=p^{\prime}$, and the second gives $\frac{1}{p^{\prime}}<\gamma$ or

$$
\frac{p-1}{p}<r
$$

For $p=p^{\prime}=2$ we get

$$
\frac{1}{2}<\gamma
$$

REMARK 3.2.

In this paragraph we would like to employ some conditions which are rather situated in between the Lipschitz and the Dini-Lipschitz conditions. These were inspired from Weiss and Zygmund [5]. Thus we prove the following theorem. 
THEOREM 3.3.

Let $f(x)$ belong to $L^{p}(R) 1<p \leq 2$ such that

$$
\| f(x+h)-\left.f(x)\right|_{p}=0\left[\frac{h^{\alpha}}{\log h^{\gamma}}\right]
$$

$0<\alpha \leq 1$ as $h+0$. Then $\hat{f} \in L^{\beta}(R)$ for

$$
\frac{p}{p+\alpha p-1}<\beta \leq p^{\prime}=\frac{p}{p^{-1}}
$$

and

$$
\frac{1}{3}<\gamma
$$

PROOF. The proof goes exactly as that of theorem (3.1) and yields

$$
\int_{1}^{x}|\hat{f}|^{\beta} d u=0[\log x]^{-\gamma \beta} x^{1-\beta-\alpha \beta+\frac{\beta}{p}}
$$

and for the right hand of this estimate to be bounded as $x \rightarrow \infty$ one must have

$$
1-\beta-\alpha \beta+\frac{\beta}{p} \leq 0
$$

and $-\gamma B<-1$

The first restriction gives the original conclusion of Titchmarsh's theorem 84 and the second gives $\frac{1}{\beta}<\gamma$. The choice $\alpha=1$ gives

$$
\|f(x+h)-f(x)\|_{p}=0\left[\frac{h}{\log h^{\gamma}}\right]
$$

and we get

$$
\frac{p}{2 p-1}<B \leq p^{\prime}
$$

and for $p=p^{\prime}=2, \gamma$ must be greater than $\frac{1}{2}$.

NOTICE 3.4 .

In [2] Titchmarsh's Theorems were proved for higher differences or equivalently for higher derivatives of $f(x)$. This indicates that if we use the conditions

$$
\begin{aligned}
& o\left[\log \left(\frac{1}{h}\right)\right]^{-1} \\
& \left\|\Delta_{h}^{r} f(x)\right\|_{p}=\quad \circ\left[\log \left(\frac{1}{h}\right)\right]^{-\gamma}
\end{aligned}
$$

or

$$
w_{p}(h) f_{(x)}^{(r)}=\quad 0\left[\frac{h^{\alpha}}{\log h^{\gamma}}\right]
$$


where

$$
\Delta_{h}^{r} f(x)=\sum_{i=0}^{r}(-1)^{r-1}\left(\begin{array}{l}
r \\
1
\end{array}\right) f(x+1 h)
$$

Then we will arrive at the same results proved in the previous theorems.

Another valid point here is that if we turn to the realm of Fourier Series of functions on $L^{p}(T)$ we will get the same conclusions except $L^{\beta}(R)$ is replaced now with the sequence space $1^{B}$ and the summation is taken over the integers $Z$. Appart from that, the definitions and the proofs are exactly the same.

4. FUNCTIONS OF SEVERAL VARIABLES.

Titchmarsh's Theorems were generalized also for functions in $L^{p}\left(R^{n}\right)$ and $L^{p}\left(T^{n}\right)$ (see [2] and [3]), without any change in the results. In contrast, we expect that for the Dini-Lipschitz functions in $L^{P}\left(R^{n}\right)$ and $L^{P}\left(T^{n}\right)$ the foregoing conclusions hold verbal1y.

To see this we would like to point out that there are two definitions for Lipschitz functions of several variables. We confine ourselves to functions in $R^{2}$ and $T^{2}$ for simplicity. Thus we introduce the following definitions.

DEFINITION 4.1 .

Let $f(x, y)$ belong to $L^{p}\left(R^{2}\right)$. Then we say that $f$ belongs to $\operatorname{Lip}\left(\alpha_{1}, p\right)$ in $x$ and to $\operatorname{Lip}\left(\alpha_{2}, p\right)$ in $y$ if

$$
\begin{gathered}
\|f(x+h, y+k)-f(x, y+k)-f(x+h, y)+f(x, y)\|_{p} \\
=0\left[h^{\left.\alpha_{1} \alpha^{\alpha_{2}}\right]}\right. \\
0<\alpha_{1}, \alpha_{2} \leq 1 .
\end{gathered}
$$

Another definition states that

$$
\|f(x+h, y+k)-f(x, y)\|_{p}=0\left[h^{\alpha_{1}}+k^{\alpha_{2}}\right]
$$

We indicates that in [2] and [3] we have employed the two definitions for functions in $L^{p}\left(R^{n}\right)$ and $L^{p}\left(T^{n}\right)$ and obtained the same conclusions of Titchmarsh's theorems. However the steps of the proofs when the first definition was employed were straight forward, where as the arguments in case of the second definition needed to be handled with special care.

In view of the previous considerations we introduce the following:

DEFINITION 4.2 .

The function $f(x, y)$ in $L^{p}\left(R^{2}\right)$ belongs the Dini-Lipschitz class if

$$
\begin{aligned}
\| f(x+h, y+k) & -f(x, y+k)-f(x+h, y)+f(x, y) \|_{p} \\
& =0\left[\log \left(\frac{1}{h}\right) \log \left(\frac{1}{k}\right)\right]^{-1}
\end{aligned}
$$

as $h, k \rightarrow 0$. 
Other classes of functions can be obtained by replacing the right hand side of this estimate by

$$
\begin{gathered}
o\left[\log \left(\frac{1}{h}\right)\right]^{-\gamma_{1}}\left[\log \left(\frac{-}{k}\right)^{1}\right]^{-\gamma} 2 \\
o\left[\frac{h^{\alpha} 1}{\log h^{\gamma_{l}}}\right]\left[\frac{k^{\alpha_{2}}}{\log k^{\gamma_{2}}}\right]
\end{gathered}
$$

and

$$
0\left[\frac{h^{\alpha_{1}}}{\log h^{\gamma_{1}}}\right]\left[\frac{k^{\alpha_{2}}}{\operatorname{Logk} k^{\gamma_{2}}}\right]
$$

respectively.

DEFINITION 4.3 .

The function $f(x, y)$ belongs to the Dini-Lipschitz class in $L^{p}\left(R^{2}\right)$ if

$$
\begin{aligned}
\|f(x+h, y+k)-f(x, y)\|_{p} & =o\left[\log \left(\frac{1}{h}\right)+\log \left(\frac{1}{k}\right)\right]^{-1} \\
& =o\left[\left(\log \left(\frac{1}{h}\right)\right)^{-1}+\left(\log \left(\frac{1}{k}\right)\right)^{-1}\right],
\end{aligned}
$$

other function classes are defined as

$$
\begin{aligned}
& 0\left[\log \left(\frac{1}{h}\right)^{-\gamma_{1}}+\log \left(\frac{1}{k}\right)^{-\gamma_{2}}\right] \\
\|f(x+h, y+k)-f(x, y)\|_{p}= & 0\left[\left(\frac{h^{\alpha_{1}}}{\log h_{1}^{\gamma_{1}}}\right)+\left(\frac{h^{\alpha_{2}}}{\log k^{\gamma_{2}}}\right)\right]
\end{aligned}
$$

We now state and prove the following theorem

THEOREM 4.4 .

Let $f(x, y)$ belong to $L^{p}\left(R^{2}\right) 1<p \leq 2$, and let

$$
\begin{array}{r}
\|f(x+h, y+k)-f(x, y+k)-f(x+h, y)+f(x, y)\|_{p} \\
=0\left[\frac{h_{1}^{\alpha_{1}}}{\log \gamma_{1}}\right]\left[\frac{k^{\alpha_{2}}}{\log \gamma_{2}}\right], 0<\alpha_{1}, \alpha_{2} \leq 1
\end{array}
$$

Then its Fourier transform $\hat{f}(u, v)$ belongs to $L^{\beta}$ where

$$
\begin{aligned}
& \frac{p}{p+\alpha_{1} p-1}<\beta \leq p^{\prime} \\
& \frac{p}{p+\alpha_{2} p-1}<\beta \leq p^{\prime}
\end{aligned}
$$


308

M. S. YOUNIS

$$
\frac{1}{\beta}<\gamma_{1}, \frac{1}{B}<\gamma_{2}
$$

PROOF .

As in the proof of theorem 84 of Titchmarsh, we obtain

$$
\int_{0}^{\frac{1}{h}} \int_{0}^{\frac{1}{k}}|u v \hat{f}|^{p^{\prime}} \operatorname{dudv}=0\left[\frac{h^{\alpha_{1}-1}}{\log h^{\gamma_{1}}}\right]\left[\frac{k^{\alpha_{2}-1}}{\log k^{\gamma_{2}}}\right]^{p^{\prime}}
$$

Now let

$$
\phi(X . Y)+\int_{1}^{X} \int_{1}^{Y}|u v \hat{f}|^{\beta} d u d v
$$

Then for $B \leq \mathrm{P}^{-}$and by the Holder Inequality we arrive at

$$
\phi(X, Y)=0\left[X^{1-\alpha_{1} \beta+\frac{\beta}{P}} \log X^{-\gamma_{1} \beta}\right]\left[Y^{1-\alpha_{2} \beta}+\frac{\beta}{P} \log Y^{-\gamma_{2} \beta}\right],
$$

so that

$$
\begin{aligned}
& \int_{1}^{X} \int_{1}^{Y}|\hat{f}|^{\beta} d u d v \\
& =0\left[X^{1-\beta-\alpha_{1} \beta+\frac{\beta}{P}}\left(\log X^{-\alpha_{1} \beta}\right)\right] x \\
& {\left[Y^{1-\beta-\alpha_{1} \beta+\frac{\beta}{P}}(\log Y)^{-\gamma^{2}{ }_{2} \beta}\right]}
\end{aligned}
$$

For the last quantity to be bounded as $\mathrm{X}, \mathrm{Y} \rightarrow \infty$ we get the required conclusions

$$
\begin{aligned}
& 1-\beta-\alpha_{1} \beta+\frac{\beta}{p} \leq 0 .-\gamma_{1} \beta<-1 \\
& 1-\beta \alpha_{2} \beta+\frac{\beta}{p} \leq 0 \\
& -\gamma_{2} \beta<-1
\end{aligned}
$$

which give

$$
\frac{p}{p+\alpha p-1}<\beta \leq p^{\prime}
$$

where $\alpha=\min \left(\alpha_{1}, \alpha_{2}\right)$

and

$$
\frac{1}{B}<\min \left(\gamma_{1},-\ddot{\gamma}_{2}\right) .
$$


We indicate that if we use the previous definition with higher differences or if we employ the Fourier Series in $L^{p}\left(T^{2}\right)$ we still get the same results of theorem 4.4 The proofs are direct and we omit them.

We hint also that the conclusion of theorem 4.4 could have been stated equivalently in the following manner that $\hat{f}(u, v) \in L^{\beta_{1}} \cap L^{\beta_{2}}$ where

$$
\begin{aligned}
& \frac{p}{p+\alpha_{1} p-1}<\beta_{i}<p^{\prime} \\
& \frac{1}{B_{i}}<\gamma_{i} \\
& i=1,2 .
\end{aligned}
$$

We conclude this section by adding that we could have used definition 4.3, and here we could have arrived at similar results in case of Fourier transform and Fourier Series, however, the proofs in this case are not so direct as in the previous cases. 5. FUNCTIONS IN $\mathrm{L}^{2}$ (R) AND $\mathrm{L}^{2}(\mathrm{~T})$.

The special case $0<\alpha<1$ and $p=2$ [1, Theorem 85] deserves some consideration in this work. Titchmarsh proved that if $f(x) \in L^{2}(R)$ then the conditions

$$
\begin{aligned}
\|f(x+h)-f(x)\|_{2}= & 0\left(h^{\alpha}\right) \\
\text { as } & h \rightarrow 0
\end{aligned}
$$

and

$$
\left[\int_{-\infty}^{-x}+\int_{x}^{\infty}\right]|\hat{f}|^{2} d u=0\left[x^{-2 \alpha}\right]
$$

as $\mathrm{X} \rightarrow \infty$ are equivalent.

In [2] and [3] we extended this theorem to higher differences of functions in $L^{2}\left(R^{n}\right)$ and $L^{2}\left(T^{n}\right)$ respectively. Here we examine the analogus situation for the Dini-Lipschitz class and start with the following.

THEOREM 5.1 .

Let $f(x) \quad L^{2}(R)$. Then the conditions

$$
\|f(x+h)-f(x)\|_{2}=o\left[\log \left(\frac{1}{h}\right)\right]^{-1}
$$

as $\mathrm{h} \rightarrow 0$,

$$
\left[\int_{-\infty}^{-x}+\int_{x}^{\infty}\right]|\hat{f}|^{2} d u=0[\log x]^{-1}
$$

as $\mathrm{X} \rightarrow \infty$ are equivalent.

PROOF .

Applying the Parseval's Identity and following Titchmarsh's proof we get in this case

$$
\int_{\frac{1}{2 h}}^{\frac{1}{h}}|\hat{f}|^{2} d u=o\left[\log \left(\frac{1}{h}\right)\right]^{-2}
$$


so that

$$
\begin{aligned}
& \int_{x}^{\infty}|\hat{f}|^{2} d u=\left[\int_{x}^{2 x}+\int_{2 x}^{4 x}+\int_{4 x}^{\gamma x} \cdots\right]|\hat{f}|^{2} d u \\
& =o\left[(\log x)^{-2}+(\log 2 x)^{-2}+(\log 4 x)^{-2}+\ldots\right] \\
& =o\left[(\log x)^{-2} 1+\left(1+\frac{\log 2}{\log X}\right)^{-1}+\left(1+\frac{2 \log 2}{\log x}\right)^{-1} \ldots\right. \\
& +\left(1+\frac{n \log 2}{\log x}\right)^{-1} \ldots .1 \\
& \text { But }\left(1+\frac{n \log 2}{\log x}\right)^{-1}=\left(\frac{\log x}{\log 2^{n} x}\right) \\
& \left(1+\frac{\log 2}{\log x}\right)^{-1}+\left(1+\frac{2 \log 2}{\log x}\right)^{-1}+\ldots \\
& +\left(1+\frac{n \log 2}{\log X}\right)^{-1} \\
& =\log x\left[\frac{1}{\log X+\log 2}+\frac{1}{\log X+2 \log 2}\right]
\end{aligned}
$$

and the series in the brackets reduces to

$$
\left[\frac{1}{\log 1}+\frac{1}{2 \log 2}+\frac{1}{3 \log 2} \ldots\right]
$$

as $\mathrm{X} \rightarrow \infty$.

Which is convergent by the comparison with the power series. Hence we arrive at last to the estimate.

$$
\int_{x}|\hat{f}|^{2} d u=o[\log x]^{-1}
$$

which proves the first assertion. The converse can be delt with in a simflar fashion. If we replace $o\left[\log \left(\frac{1}{h}\right)\right]_{h^{\alpha}}^{-1}$ by $o\left[\log \left(\frac{l}{h}\right)\right]^{-\gamma}$ we get the same result. But now let us examine the estimate $0\left[\frac{h^{\alpha}}{\log h^{\beta}}\right] 0<\alpha<1$. We state the following theorem.
THEOREM 5.2. Let $f(x) \in L^{2}(R)$. Then the conditions

$$
\|f(x+h)-f(x)\|_{2}=0\left(\frac{h^{\alpha}}{\log h^{\beta}}-\right)
$$


as $\mathrm{h}+0$ and

$$
\left[\int_{-\infty}^{-X}+\int_{X}^{\infty}\right]|\hat{f}|^{2} d u=0\left[x^{-2 \alpha}(\log h)^{-2 \beta}\right]
$$

as $X \rightarrow \infty$ are equivalent and in fact if $\beta>\frac{1}{2}$ the right hand side of the last estimate can be replaced with $0[\mathrm{X}]$.

We shall not prove this in detail since the main trend of the proof is quite clear. In comparision with the previous theorem, thus

$$
\begin{aligned}
& \int_{x}^{\infty}|\hat{f}|^{2} d u=0\left[x^{-2 \alpha}(\log x)^{-2 \beta}+\left(x^{-2 \alpha}\right)\left(\log 2 x^{-2 \beta}\right) \ldots\right] \\
& =0\left[x^{-2 \alpha}[\log x]^{-2 \beta}\right]\left[1+(2)^{-2 \alpha}\left[1+\frac{\log 2}{\log x}\right]^{-2 \beta}\right. \\
& \left.+\left(2^{n}\right)^{-2 \alpha}\left[1+\frac{n \log 2}{\log x}\right]^{-2 \beta} \ldots\right] \\
& \left.+\left(2^{n}\right)^{-2 \alpha}\left[1+\frac{2 \log 2}{\log x}\right]^{-2 \beta} \ldots\right]
\end{aligned}
$$

Now the terms in the brackets

$$
\left(1+\frac{k \log 2}{\log X}\right)^{-2 B}
$$

$k=1,2 \ldots n$

are all bounded by $\left[1+\frac{\log 2}{\log X}\right]^{-2 B}$

$$
=\left[\frac{\log x}{\log 2+\log x}\right]^{2}
$$

which tends to 1 as $\mathrm{X} \rightarrow \infty$.

So that we are left with

$$
\int_{X}^{\infty}|\hat{f}|^{2} d u=0\left[x^{-2 \alpha}(\log x)^{-2 \beta}\right]\left[1+2^{-2 \beta}+2^{-4 \alpha}+\ldots\right]
$$

which proves the first part of the theorem. The converse can be carried in exactly the same manner as in Titchmarsh's theorem 85 and the proof is complete. Here again the choice $\beta>\frac{1}{2}$ reduces the conclusions of the theorem to the original case. i.e.

$$
\left[\int_{-\infty}^{-x}+\int_{x}^{\infty}\right]|\hat{f}|^{2} d u=0\left[x^{-2 \alpha}\right]
$$


6. CONCLUDING REMARKS.

The treatments in the previous section convince us that for the various types of Dini-Lipschitz functions in $L^{2}(T)$. $L^{2}\left(R^{n}\right)$, and $L^{2}\left(T^{n}\right)$ the analysis can be carried almost without much difficulty, however, even the statements of the results in case of $L(R)$ and $L(T)$ would be fairly complicated.

We conclude finally that Titchmarsh's theorems [especially Theorem 85] were extended in [3] as well as in various papers in the Literature to other groups such as the 0-dimensional, the finite dimensional and compact Lie groups. We indicate that in a forth-coming paper we shall be dealing with the present subject along those directions.

REFERENCES

1. TITCHMAKSH, E.C. Theory of Fourier Integral, 2nd Ed., Oxford Univ. Press, 1948.

2. YOUNIS, M.S. Fourier Transforms in $\mathrm{L}^{\mathrm{p}}$ Spaces, M. Phil. Thesis, Chelsea College, London, 1970

3. YOUNIS, M.S. Fourler Transforms of Lipschitz Functions on Compact Groups. Ph.d. Thes is McMaster University, Hamilton, Ontario, Canada, 1974.

4. KROVOKIN, P.P. Linear Operators and Approximation Theory. International Monographs on Advanced Mathematics and Physics, 1960.

5. WEISS, M. and ZYGMUND, A. A Note on Smooth Functions, Indag. Math. 2 (1959), 52-58. 


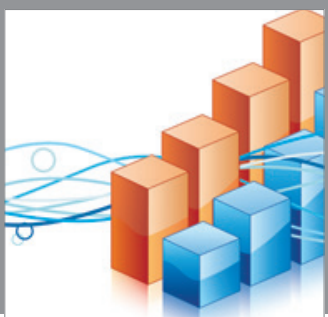

Advances in

Operations Research

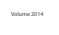

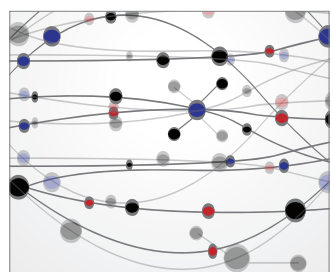

\section{The Scientific} World Journal
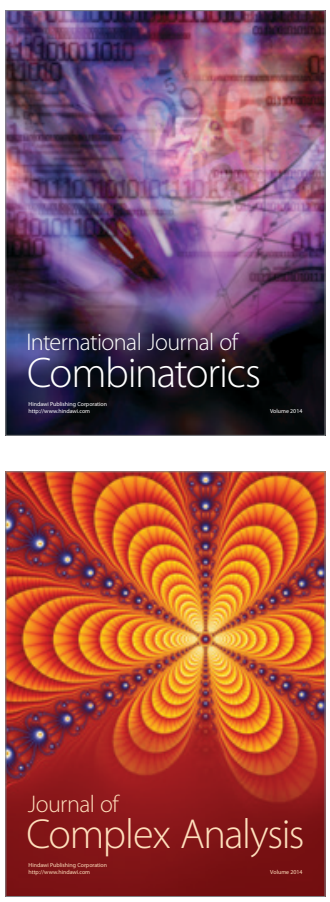

International Journal of

Mathematics and

Mathematical

Sciences
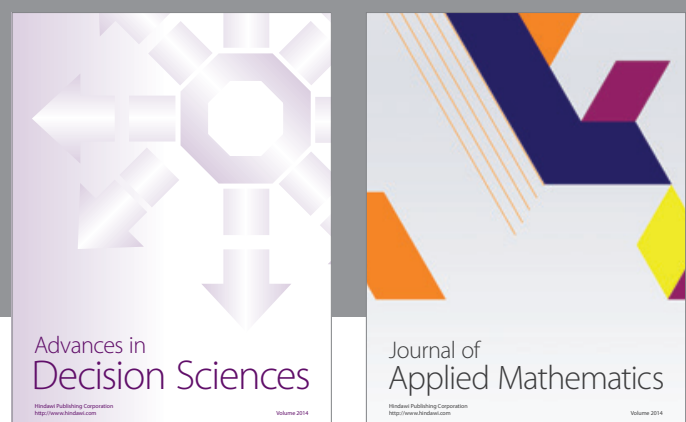

Journal of

Applied Mathematics
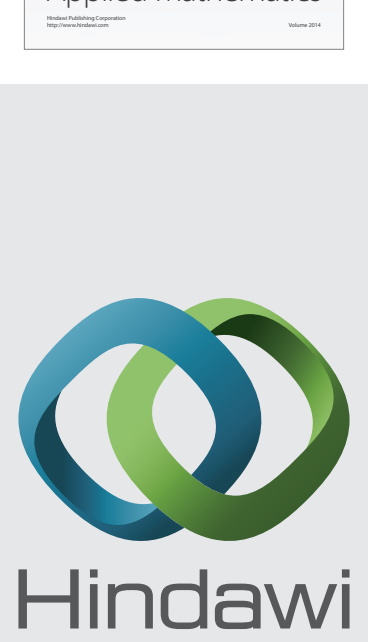

Submit your manuscripts at http://www.hindawi.com
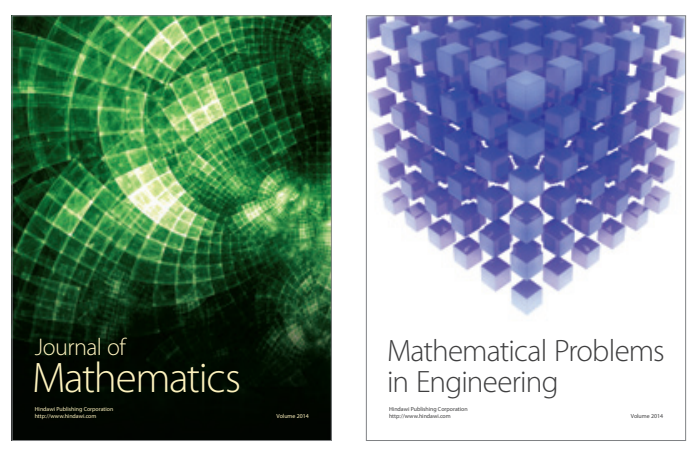

Mathematical Problems in Engineering
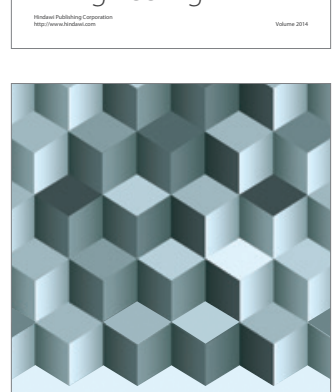

Journal of

Function Spaces
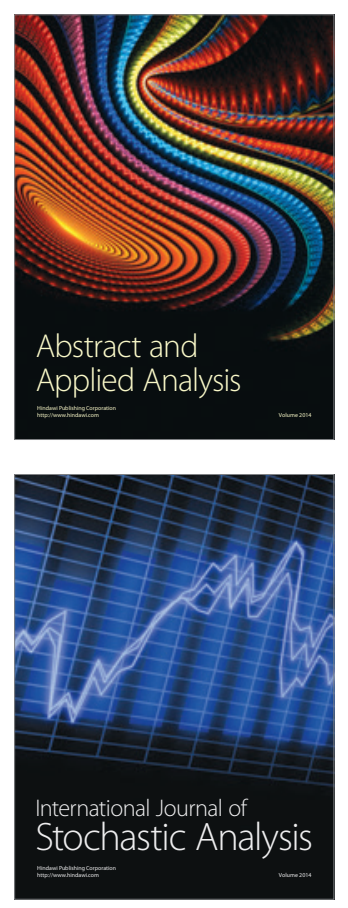

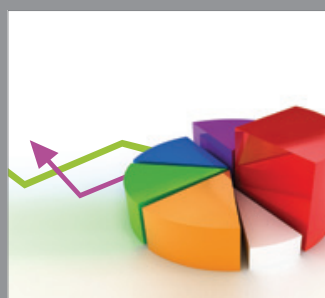

ournal of

Probability and Statistics

Promensencen
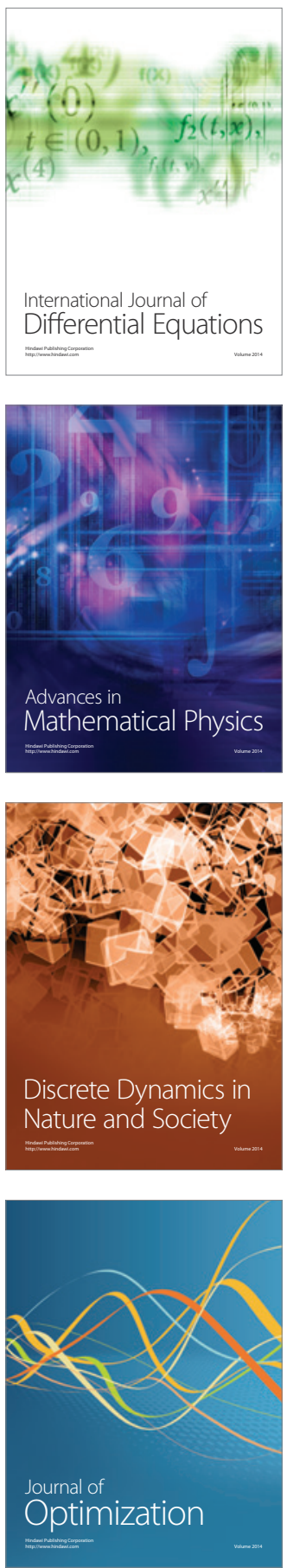